\title{
SiG12D LODER
}

National Cancer Institute

\section{Source}

National Cancer Institute. siG12D LODER. NCI Thesaurus. Code C95772.

A proprietary, miniature biodegradable polymeric matrix containing small-interfering RNAs for the mutated KRAS oncogene, KRASG12D, (siG12D), with potential antitumor activity. Upon intratumoral injection, this siG12D is released locally, thereby preventing translation of KRAS proteins and potentially inhibiting growth of tumor cells overexpressing KRAS. KRAS, a member of the small GT Pase superfamily, is mutated in over $90 \%$ of human pancreatic ductal adenocarcinomas (PDAC) and is associated with tumor cell proliferation and reduced survival. 